Pacific Journal of Mathematics

A NOTE ON TAMELY RAMIFIED EXTENSIONS OF RINGS 


\title{
A NOTE ON TAMELY RAMIFIED EXTENSIONS OF RINGS
}

\author{
M. L. BROWN
}

Buhler gave a criterion for a class of finite free extensions of discrete valuation rings to be tamely ramified 1-dimensional regular rings. In this note, we extend this criterion to finite free extensions of general local rings and, in the final section, indicate the extension to schemes.

1. Introduction. To set the notation, let $A$ be a noetherian local ring of Krull dimension $n$ and let $A \rightarrow B$ be a finite free extension of rings; denote by $\mathfrak{D}_{B / A}$ the discriminant of this extension, defined as $\operatorname{det}\left[\operatorname{tr}\left(b_{i} b_{j}\right)\right]$ where $b_{1}, \ldots, b_{m}$ is a free basis of $B$ over $A$ and $\operatorname{tr}: B \rightarrow A$ denotes the trace morphism. Let $\mathfrak{m}_{A}$ be the maximal ideal of $A$ and define a function $\nu_{\mathfrak{m}_{A}}$ on $A$ by $\nu_{\mathfrak{m}_{A}}(x)=r$ where $r$ is the largest integer with $x \in \mathfrak{m}_{A}^{r}$ and $\nu_{\mathfrak{m}_{A}}(0)=\infty$. Note that $\nu_{\mathfrak{m}_{A}}$ is a valuation if $\operatorname{gr}_{A}\left(\mathfrak{m}_{A}\right)$ has no zero divisors, in particular if $A$ is regular [2].

If $\mathfrak{n}_{1}, \ldots, \mathfrak{n}_{s}$ are the maximal ideals of $B$ lying over $\mathfrak{m}_{A}$ define the ramification index $e_{\mathfrak{n}_{1} / \mathfrak{m}_{A}}$ to be $l_{B_{\mathrm{n}_{t}}}\left(B_{\mathfrak{n}_{t}} / \mathfrak{m}_{A} B_{\mathfrak{n}_{t}}\right)$ where $l_{B}(M)$ denotes the length (of a composition series) of the artin $B$-module $M$. If $A$ is a discrete valuation ring, the $e_{\mathfrak{n}_{l} / \mathrm{m}_{A}}$ clearly coincide with the usual ramification indices of algebraic number theory. Recall that the embedding dimension $\operatorname{ed}(B)$ of the semi-local ring $B$ is $\max \operatorname{dim}_{\kappa\left(\mathfrak{n}_{l}\right)} \mathfrak{n}_{i} / \mathfrak{n}_{i}^{2}$ where $\mathfrak{n}_{l}$ runs through all maximal ideals of $B$. With the above notation the main result of this paper is:

THEOREM 1. If $A$ is regular (resp. $\mathrm{gr}_{A}\left(\mathrm{~m}_{A}\right)$ has no zero divisors) and if $B=A[X] /\langle f(X)\rangle$ where $f(X)$ is a monic polynomial and $\kappa(\mathfrak{m}) \rightarrow \kappa\left(\mathfrak{n}_{i}\right)$ is separable for all $i=1, \ldots, s$, then

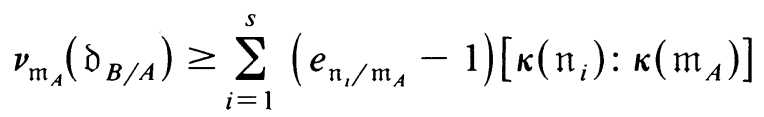

with equality if and only if (resp. only if) $\operatorname{ed}(B)=\operatorname{ed}(A)$ and $B$ is tamely ramified over $A$ in that $p \nmid e_{\mathfrak{n}_{l} / \mathfrak{m}_{A}}$ for all $i$, where $p$ is the characteristic of $\kappa\left(\mathrm{m}_{A}\right)$. 
2. Proof of Theorem 1. We begin by some reductions. Observe that the conditions and conclusions of the theorem remain unchanged on base change by the $\mathrm{m}_{A}$-adic completion of $A: A^{\wedge} \rightarrow B \otimes_{A} A^{\wedge} \cong B^{\wedge}$ so we may assume $A$ is complete. Thus $B$ is a product of local rings $\Pi B_{i}$ where each $A \rightarrow B_{1}$ satisfies the conditions of Theorem 1. Since $\nu_{\mathrm{m}_{A}}\left(\grave{D}_{B / A}\right)=$ $\Sigma_{l} \nu_{\mathrm{m}_{A}}\left(\mathfrak{D}_{B_{l} / A}\right)$, it is easy to see that it is enough to prove Theorem 1 when $B$ is local with maximal ideal $\mathfrak{m}_{B}$, say.

Let $e$ be the ramification index of $B$ over $A$ and $a_{1}, \ldots, a_{n}$ form a basis of the cotangent space $\mathrm{m}_{A} / \mathrm{m}_{A}^{2}$ over $\kappa\left(\mathrm{m}_{A}\right)$. There is a monic polynomial $g \in A[X]$ with $f=g^{e}+\sum_{l} a_{l} h_{l}$ where $h_{l} \in A[X]$ for all $i$. Letting $R(p(X), q(X))$ denote the resultant of the polynomials $p$ and $q$ (see [3] or [5] for the properties of resultants we will use), then

$$
\mathfrak{D}_{B / A}=R\left(f, f^{\prime}\right)=R\left(f, e g^{e-1} g^{\prime}+\sum_{i} a_{l} h_{i}^{\prime}\right) .
$$

If $p \mid e$ where $p=\operatorname{char} \kappa\left(\mathfrak{m}_{A}\right)$, then $e \in \mathfrak{m}_{A}$ and so

$$
\begin{aligned}
\nu_{\mathrm{m}_{A}}\left(\delta_{B / A}\right) & =\nu_{\mathrm{m}_{A}}\left(R\left(f, \mathrm{eg}^{e-1} g^{\prime}+\sum_{l} a_{\imath} h_{i}^{\prime}\right)\right) \\
& \geq \text { degree } f=e\left[\kappa\left(\mathrm{m}_{B}\right): \kappa\left(\mathrm{m}_{A}\right)\right] .
\end{aligned}
$$

This completes the proof for the case of wild ramification.

Assume from now on that $e \notin m_{A}$. Since $f^{\prime} \equiv e g^{\prime} g^{e-1} \bmod m_{A}$ and $\kappa\left(\mathrm{m}_{A}\right) \rightarrow \kappa\left(\mathrm{m}_{B}\right)$ is separable, eg and $g^{e-1}$ are relatively prime in $\kappa\left(\mathrm{m}_{A}\right)[X]$. Thus by Hensel's lemma

$$
f^{\prime}=\left(e g^{\prime}+\sum_{i} a_{\imath} p_{i}\right)\left(g^{e-1}+\sum_{i} a_{l} q_{l}\right)
$$

where $p_{i} q_{i} \in A[X]$ with $\operatorname{deg}\left(p_{t}\right)<\operatorname{deg}\left(g^{\prime}\right), \operatorname{deg}\left(q_{i}\right)<\operatorname{deg} g^{e-1}$ for all $i$.

Since $\nu_{\mathrm{m}_{A}}\left(R\left(g^{e}, e g^{\prime}\right)\right)=e \nu_{\mathrm{m}_{A}}\left(R\left(g, g^{\prime}\right)\right)=0$ we have

$$
\nu_{\mathrm{m}_{A}}\left(R\left(f, e g^{\prime}+\sum a_{i} p_{i}\right)\right)=0 \text {. }
$$

Thus

$$
\begin{aligned}
\nu_{\mathrm{m}_{A}}\left(\mathfrak{D}_{B / A}\right) & =\nu_{\mathrm{m}_{A}}\left(R\left(f, \mathrm{eg}^{\prime}+\sum_{i} a_{l} p_{l}\right)\right)+\nu_{\mathrm{m}_{A}}\left(R\left(f, g^{e-1}+\sum_{l} a_{l} q_{l}\right)\right) \\
& =\nu_{\mathrm{m}_{A}}\left(R\left(f, g^{e-1}+\sum_{l} a_{i} q_{l}\right)\right)
\end{aligned}
$$

we conclude that if $e=1$ then $\nu_{\mathfrak{m}_{A}}\left(\mathfrak{D}_{B / A}\right)=0$ and $\mathfrak{m}_{A} B=\mathfrak{m}_{B}$ proving the theorem for the unramified case $e=1$. 
Assume from now on that $e \geq 2$ and put $r=g^{e-1}+\Sigma_{l} a_{i} q_{i}$. Then

$$
\begin{aligned}
\nu_{\mathfrak{m}_{A}}\left(\grave{D}_{B / A}\right) & =\nu_{\mathfrak{m}_{A}}(R(f-g r, r))=\nu_{\mathrm{m}_{A}}\left(R\left(\sum_{i} a_{i}\left(h_{i}-g q_{i}\right), r\right)\right) \\
& \geq \operatorname{deg} r=(e-1)\left[\kappa\left(\mathrm{m}_{B}\right): \kappa\left(\mathrm{m}_{A}\right)\right]
\end{aligned}
$$

and equality holds if and only if (resp. only if)

$$
I=\left\langle h_{1}-g q_{1}, \ldots, h_{n}-g q_{n}, r\right\rangle \kappa\left(\mathfrak{m}_{A}\right)[X]=\kappa\left(\mathrm{m}_{A}\right)[X]
$$

by Lemma 1 below. This completes the proof, for $I=\kappa\left(\mathrm{m}_{A}\right)[X]$ if and only if some $h_{i}$ is invertible in $\kappa\left(\mathfrak{m}_{B}\right)$ and so if and only if $\sum a_{i} h_{i}=f-g^{e}$ $\equiv 0 \operatorname{mod~} \mathrm{m}_{B}^{2}$ gives a non-trivial linear relation between the $a_{i}$ 's and $g$ in $\mathfrak{m}_{B} / \mathfrak{m}_{B}^{2}$.

LEMMA 1. If $A$ is a regular local ring (resp. a local ring) and $a_{1}, \ldots, a_{n} a$ basis of $\mathrm{m}_{A}$ and $p_{0}, \ldots, p_{n} \in A[X]$ with $p_{0}$ monic, then

$$
R\left(\sum_{i=1}^{n} a_{i} p_{i}, p_{0}\right) \notin \mathrm{m}_{A}^{1+\operatorname{deg} p_{0}}
$$

if and only if (resp. only if ) $p_{0}, \ldots, p_{n}$ are coprime in $\kappa\left(\mathrm{m}_{A}\right)[X]$.

Proof. If $A$ is an arbitrary local ring, let $m \in A[X]$ be a monic polynomial with residue in $\kappa\left(\mathfrak{m}_{A}\right)[X]$ the highest common factor of $p_{0}, \ldots, p_{n}$. Then for some $q_{i} \in A[X]$ with $q_{0}$ monic and $\operatorname{deg} m q_{0}=\operatorname{deg} p_{0}$, $R\left(\sum_{i} a_{i} p_{i}, p_{0}\right) \equiv R\left(m \sum a_{i} q_{i}, m q_{0}\right) \bmod \mathrm{m}_{A}^{\operatorname{deg} p_{0}+1}$ so if $\operatorname{deg} m \geq 1$, $R\left(\sum a_{i} p_{i}, p_{0}\right) \in \mathfrak{m}_{A}^{\operatorname{deg} p_{0}+1}$ as required.

Conversely, if $A$ is regular $\operatorname{gr}_{A}\left(\mathrm{~m}_{A}\right)$ is a polynomial ring $\kappa\left(\mathfrak{m}_{A}\right)\left[X_{1}, \ldots, X_{n}\right]$ [2], with the usual grading, so that monomials of total degree $d$ in $a_{1}, \ldots, a_{n}$ are linearly independent in $A / \mathrm{m}_{A}^{d+1}$. Since $R\left(\sum_{t} a_{i} p_{i}, p_{0}\right)$ is a homogeneous polynomial of degree deg $p_{0}$ in the $a_{i}$ in $A, \nu_{\mathrm{m}_{A}}\left(R\left(\Sigma_{l} a_{l} p_{i}, p_{0}\right)\right)=1+\operatorname{deg} p_{0}$ if and only if $R\left(\sum Z_{i} p_{i}, p_{0}\right)$ is the zero polynomial in the ring $\kappa\left(\mathrm{m}_{A}\right)\left[Z_{1}, \ldots, Z_{n}\right]$ where the $Z_{l}$ 's are indeterminates.

Now if $p_{i}$ are coprime in $\kappa\left(\mathfrak{m}_{A}\right)[X]$ then $\sum_{l=0}^{n} c_{i} p_{i} \equiv 1 \bmod \mathfrak{m}_{A}$ for some $c_{i} \in A[X]$. Thus

$$
\nu_{\mathrm{m}_{A}}\left(R\left(\sum_{i=1}^{n} c_{i} p_{i}, p_{0}\right)\right)=\nu_{\mathrm{m}_{A}}\left(R\left(\sum_{i=0}^{n} c_{i} p_{\imath}, p_{0}\right)\right)=0
$$

so $R\left(\sum Z_{i} p_{i}, p_{0}\right)$ is not the zero polynomial in $\kappa\left(\mathfrak{m}_{A}\right)[Z]$ proving the lemma. 
3. The obstruction for non-regular rings. Throughout this section the local ring $A$ is assumed to have no zero divisors in $\operatorname{gr}_{A}\left(\mathrm{~m}_{A}\right)$.

For regular rings, Theorem 1 gives a necessary and sufficient numerical criterion for $A \rightarrow A[X] /\langle f(X)\rangle$ to be tamely ramified with $\operatorname{ed}(f)=$ $\operatorname{ed}(A)$. The failure of this criterion to be necessary for non-regular rings is examined in this section; we will see that the obstruction lies in the equations defining the tangent cone $\operatorname{gr}_{A}\left(\mathfrak{m}_{A}\right)$. Indeed, we construct a cohomology group $H^{2}\left(C_{g}^{*}\right)$ so that the numerical criterion is necessary and sufficient for all polynomials with a fixed reduction $g \bmod \mathrm{m}_{A}$, say, if and only if $H^{2}\left(C_{g}^{*}\right)$ is isomorphic to the vector space of homogeneous equations defining the tangent cone of degree equal to that of $g(X)$.

In the sense of Hilbert schemes classifying polynomials over $A$, this failure is not exceptional: "ailmost all" tamely ramified polynomials $f(X)$ over a non-regular ring $A$ with $\nu(\operatorname{discr} f)>\Sigma_{i}\left(e_{l}-1\right)\left[\kappa\left(\mathfrak{n}_{l}\right): \kappa\left(\mathrm{m}_{A}\right)\right]$ have $\operatorname{ed}(f(X))=\operatorname{ed}(A)$;

Nevertheless, for polynomials which are unramified or totally ramified or have degree $\leq 3$, the numerical criterion is necessary and sufficient over arbitrary local rings.

Fix a monic polynomial $g(X)=X^{m}+\sum_{l=0}^{m-1} \bar{b}_{i+1} X^{\imath}$ in $\kappa\left(\mathrm{m}_{A}\right)[X]$ and let $b_{l} \in A$ be elements with residue $\bar{b}_{l}$ for all $i$. Then $g(X)$ factorizes as $\Pi_{l} \bar{g}_{l}(X)^{e_{l}}$ over $\kappa\left(m_{A}\right)$ where we assume $\bar{g}_{l}(X)$ are distinct separable polynomials over $\kappa\left(\mathrm{m}_{A}\right)$.

The Hilbert scheme $H_{g}=\operatorname{Spec} A\left[X_{1}, \ldots, X_{m}\right]_{\left\langle X_{1}-b_{1}, \ldots, X_{m}-b_{m}, \mathrm{~m}_{A}\right\rangle}$ classifies the monic polynomials with reduction $g \bmod m_{A}$ in that there is a bijection:

$H_{g}(\operatorname{Spec} A) \stackrel{\sim}{\rightarrow}\{$ Monic polynomials $f(X)$ over $A$

$$
\text { with } \left.f(X) \equiv g(X) \bmod m_{A}\right\}
$$

given by

$$
\left\{A\left[X_{1}, \ldots, X_{m}\right]_{\mathfrak{n}} \rightarrow A: X_{\imath} \mapsto c_{i}+b_{i}\right\} \rightarrow X^{m}+\sum_{i=0}^{m-1}\left(c_{i+1}+b_{\imath+1}\right) X^{\imath} .
$$

Let $T$ be the tangent cone of Spec $A$, by definition $T=\operatorname{Proj} \operatorname{gr}_{A}\left(\mathfrak{m}_{A}\right)$ where $\operatorname{gr}_{A}\left(\mathrm{~m}_{A}\right)=\bigoplus_{l=0}^{\infty} \mathrm{m}_{A}^{l} / \mathrm{m}_{A}^{i+1}$; fix a basis, once and for all, $a_{1}, \ldots, a_{n}$ of $\mathfrak{m}_{A}$ so that $n=\operatorname{ed}(A)$. Let $T^{\prime}=\mathbf{A}_{\kappa}^{n m} \times_{\kappa} T$ where $\kappa=\kappa\left(\mathfrak{m}_{A}\right)=A / \mathfrak{m}_{A}$ and $\mathbf{A}_{\kappa}^{n m}=\operatorname{Spec} \kappa\left[X_{i j}: 1 \leq i \leq m, 1 \leq j \leq n\right]$ is affine $n m$-space over $\kappa$. Regarding Spec $\kappa$ as a $T$-scheme, via projection onto the 1 st component $\mathrm{gr}_{A}\left(\mathrm{~m}_{A}\right) \rightarrow A / \mathfrak{m}_{A}=\kappa$, there is a bijection:

$$
H_{g}\left(\operatorname{Spec} A / \mathrm{m}_{A}^{2}\right) \stackrel{\sim}{\rightarrow} T-\operatorname{sch}\left(\operatorname{Spec} \kappa, T^{\prime}\right)
$$


given by

$$
\left\{f: X_{i} \rightarrow c_{i}+b_{i}\right\} \rightarrow\left\{X_{i j} \rightarrow x_{i j} \in \kappa \text { all } i, j \text { where } c_{i} \equiv \sum_{j=1}^{n} a_{J} x_{\imath J} \bmod \mathrm{m}_{A}^{2}\right\} \text {. }
$$

Denote by * the composite of the maps:

$$
H_{g}(\operatorname{Spec} A) \stackrel{\text { natural }}{\rightarrow} H_{g}\left(\operatorname{Spec} A / \mathrm{m}_{A}^{2}\right) \stackrel{\sim}{\rightarrow} T-\operatorname{sch}\left(\operatorname{Spec} \kappa, T^{\prime}\right) .
$$

Proposition 1. (1) The integer $s=\Sigma_{i}\left(e_{i}-1\right)\left[\kappa\left(\mathfrak{n}_{i}\right): \kappa\left(\mathrm{m}_{A}\right)\right]$ is the same for all polynomials in $H_{g}(\operatorname{Spec} A)$.

(2) There are closed subschemes $V \supset V^{\prime}$ of $T^{\prime}$ so that for any $h \in$ $H_{g}(\operatorname{Spec} A)$ with associated polynomial $f(X)$,

(a) $\nu_{\mathrm{m}_{A}}(\operatorname{discr} f(X))>s$ if and only if $h^{*} \in T$-sch-(Spec $\left.\kappa, V\right)$,

(b) $\operatorname{ed}(f(X))>\operatorname{ed}(A)$ if and only if $h^{*} \in T-\operatorname{sch}\left(\operatorname{Spec} \kappa, V^{\prime}\right)$.

(3) $V$ is a proper closed subscheme of $T$ if and only if all polynomials in $H_{g}(\operatorname{Spec} A)$ are tamely ramified.

\section{Proof. (1) Clear.}

(2) Recall $g(X)$ factorises as $\Pi_{i} \bar{g}_{i}^{e_{i}}$ in $\kappa[X]$ and choose representative monic polynomials $g_{i}(X) \in A[X]$ with residue $\bar{g}_{l}(X) \bmod \mathrm{m}_{A}$ for all $i$.

As in the proof of Theorem 1, it is not difficult to see that $\nu_{\mathrm{m}_{A}}(\operatorname{discr} f(X))>s$ if and only if

$$
\nu_{\mathrm{m}_{A}}\left(R\left(f(X), \sum_{i} e_{l} g_{l}^{\prime} g_{i}^{e_{i-1}} \prod_{j \neq i} g_{J}^{e_{J}}\right)\right)>s .
$$

Now

$$
R\left(f(X), \sum_{i} e_{i} g_{i}^{\prime} g_{i}^{e_{i}-1} \prod_{j \neq i} g_{j}^{e_{j}}\right)=R\left(f, \prod_{i} g_{l}^{e_{i}-1}\right) R\left(f, \sum_{i} e_{\imath} g_{l}^{\prime} \prod_{j \neq i} g_{j}\right)
$$

as in the proof of Theorem $1, \nu_{\mathrm{m}_{A}}\left(R\left(f(X), \Sigma_{i} e_{i} g_{i}^{\prime} \Pi_{j \neq l} g_{j}\right)\right)=0$ if and only if $p$ does not divide $e_{i}$ for all $i$ where $p$ is the characteristic of $\kappa\left(\mathfrak{m}_{A}\right)$.

It follows from Theorem 1 that if $p \mid e_{l}$ for some $e_{l}$ then $\nu_{\mathfrak{m}_{A}}(\operatorname{discr} f)>s$ for any $f$ with reduction $g$ so for this wildly ramified case $V=T^{\prime}$ has the required properties. If now $p \nmid e_{i}$ for all $i$ then $\nu_{\mathrm{m}_{A}}(\operatorname{discr} f(X))>s$ if and only if $\nu_{\mathrm{m}_{A}}\left(R\left(f, g_{j}\right)\right)>\operatorname{deg} g_{j}(X)$ for some $j$. Putting

$$
f(X)=X^{m}+\sum_{i=0}^{m-1}\left(b_{\imath}+\sum_{j=1}^{n} a_{j} X_{\imath J}\right) X^{i},
$$

with the notation as previously, $f(X)$ is the general polynomial of $H_{g}(\operatorname{Spec} A) ; R\left(f(X), g_{j}(X)\right)$ is a homogeneous polynomial in the $X_{l j}$ 's of 
degree $\operatorname{deg} g_{j}(X)$. Moreover, the coefficient of each monomial in the $X_{l j}$ 's is a monomial in the $a$,'s of degree $\operatorname{deg} g_{j}(X)$. Let $p_{k}\left(X_{i,}\right.$ : all $\left.i j\right)$ be the polynomial $R\left(f(X), g_{k}(X)\right)$-regarded as an element of $\operatorname{gr}\left(\mathrm{m}_{A}\right)\left[X_{t j}\right]$ of degree $\operatorname{deg} g_{k}$ and put $p\left(X_{l j}\right)=\Pi_{k} p_{k}\left(X_{i j} \text { : all } i, j\right)^{e_{k}}$. The ideal of $\operatorname{gr}\left(\mathrm{m}_{A}\right)\left[X_{i j}\right.$ : all $\left.i, j\right]$ generated by $p\left(X_{t}\right)$ clearly defines the closed subscheme $V$ of $T^{\prime}$.

(2b) With the notation above, let $f^{*}(X)$ be a polynomial from $H_{g}(\operatorname{Spec} A)$ then $f^{*}(X)=\Pi_{l} g_{l}^{e_{t}}+\sum_{l=1}^{n} a_{l} p_{i}(X)$ for some $p_{\imath}(X) \in A[X]$. We assert $\operatorname{ed}\left(f^{*}(X)\right)=\operatorname{ed}(A)$ if and only if $\Pi_{l} g_{i}^{e_{i}}, p_{1}(X), \ldots, p_{n}(X)$ have no common factor in the residue ring $\kappa\left(\mathrm{m}_{A}\right)[X]$. For, without loss of generality $A$ is complete as in the proof of Theorem 1, so $f^{*}(X)=$ $\Pi_{l}\left(g_{i}^{e_{i}}+\sum_{j=1}^{n} a_{j} p_{\imath j}(X)\right)$ for some polynomials $p_{\imath j}(X) \in A[X]$ by Hensel's lemma. By the proof of Theorem 1 and Lemma $1, \operatorname{ed}\left(f^{*}(X)\right)=\operatorname{ed}(A)$ if and only if $g_{l}(X), p_{l 1}(X), \ldots, p_{i n}(X)$ have no common factor in $\kappa\left(\mathfrak{m}_{A}\right)[X]$ for all $i$. The assertion easily follows on expanding the product for $f^{*}(X)$.

For a general polynomial $f(X)$ in $H_{g}(\operatorname{Spec} A$ ) put, as before, $f(X)=$ $X^{m}+\Sigma_{i}\left(b_{l}+\sum_{j} a_{j} X_{l j}\right) X^{i}$. Let $f^{*}(X)$ denote the specialisation of $f(X)$ under $X_{l j} \rightarrow x_{i j} \in \kappa$, then $\operatorname{ed}\left(f^{*}(X)\right)=\operatorname{ed}(A)$ if and only if $\Pi_{l} g_{l}^{e_{t}}$, $\sum_{i} x_{i} X^{i}, j=1, \ldots, n$, have no common factor in $\kappa\left(\mathrm{m}_{A}\right)[X]$. Introducing arbitrary parameters $Z_{1}, \ldots, Z_{n}$, then $\operatorname{ed}\left(f^{*}(X)\right)>\operatorname{ed}(A)$ if and only if, by Lemma $1, R\left(g(X), \sum_{j=1}^{n} Z_{j} \Sigma_{l} x_{l} X^{l}\right)$ is the zero polynomial, regarded as a polynomial in $\kappa\left(\mathrm{m}_{A}\right)\left[Z_{1}, \ldots, Z_{n}\right]$ by taking it $\bmod \mathrm{m}_{A}^{\mathrm{deg} g+1}$.

Thus $R\left(g(X), \sum_{J=1}^{n} Z_{j} \sum_{i=0}^{m} X_{l J} X^{l}\right) \bmod \mathrm{m}_{A}^{1+\operatorname{deg} g}$ is a homogeneous polynomial of degree $\operatorname{deg} g(X)=m$ in the $Z_{l}$, assuming it is non-zero.

Write $R\left(g(X), \sum_{l j} Z_{J} X_{i j} X^{i}\right) \equiv \sum Z_{\mathrm{i}} q_{\mathrm{i}}\left(X_{l j}\right) \bmod \mathrm{m}_{A}^{1+\operatorname{deg} g}$ where $Z_{\mathbf{i}}$ runs over all monomials in $Z_{l}$ of degree $m$ and $q_{\mathrm{i}}\left(X_{i j}\right) \in \kappa\left(\mathfrak{m}_{A}\right)\left[X_{i j}\right.$ : $0 \leq i \leq m-1,1 \leq j \leq n]$ is an homogeneous polynomial of degree $m$. Thus ed $\left(f^{*}(X)\right)>\operatorname{ed}(A)$ if and only if $q_{\mathrm{i}}\left(x_{i j}\right)=0$ for all $\mathbf{i}$.

Let $V^{\prime}$ be the closed subscheme of $T^{\prime}$ defined by the ideal $<q_{\mathrm{i}}\left(X_{i j}\right)$ : all $\mathbf{i}>$, then clearly $V^{\prime}$ has the required properties.

From the above proof we deduce:

COROllaRY 1. (1) Either $V=T^{\prime}$ or $V$ is a union of hypersurfaces of $T^{\prime}$ of degree $t_{k}=\left[\kappa\left(\mathfrak{n}_{k}\right): \kappa\left(\mathrm{m}_{A}\right)\right]$, with multiplicity $e_{k}$, for all $k$, and is defined by an homogeneous equation $\Pi_{k} f_{k}\left(X_{i j} \text { : all } i j\right)^{e_{k}}=0$ of degree $m=\operatorname{deg} g(x)$ with coefficients of $X_{l j}$ in $f_{k}$ homogeneous polynonials in $a_{1}, \ldots, a_{n}$ of degree $t_{k}$.

(2) $V^{\prime}$ is defined in $T^{\prime}$ by most $\left(\begin{array}{c}n-1 \\ n-1\end{array}\right)$ equations of degree $m$ in the variables $X_{i j}$ and with coefficients in $\kappa\left(\mathrm{m}_{A}\right)$. 
We relate the equations defining $V, V^{\prime}$ to those defining the tangent cone $T$ in its embedding $T \rightarrow \mathbf{P}_{k}^{n}$ given by the very ample sheaf $O_{T}(1)$. Let $S^{m}\left(\mathfrak{m}_{A} / \mathfrak{m}_{A}^{2}\right)$ denote the $m$ th symmetric power of $\mathfrak{m}_{A} / \mathfrak{m}_{A}^{2}$ and let $K_{m}$ be the kernel of the natural map $S^{m}\left(\mathfrak{m}_{A} / \mathfrak{m}_{A}^{2}\right) \stackrel{k}{\rightarrow} \mathfrak{m}_{A}^{m} / \mathfrak{m}_{A}^{m+1}$; thus $K_{m}$ is the set of "equations of degree $m$ defining $T$ ".

Suppose $g$ is tamely ramified and let $m=\operatorname{deg} g(x)$ and $s=$ $\Sigma_{i}\left(e_{i}-1\right)\left[\kappa\left(\mathrm{n}_{i}\right): \kappa\left(\mathrm{m}_{A}\right)\right]$, then there is a complex $C^{\circ}$ :

$$
0 \rightarrow T-\operatorname{sch}\left(\kappa, V^{\prime}\right) \stackrel{i}{\rightarrow} T-\operatorname{sch}(\kappa, V) \stackrel{j}{\rightarrow} S^{m}\left(\mathfrak{m}_{A} / \mathfrak{m}_{A}^{2}\right) \stackrel{k}{\rightarrow} \mathfrak{m}_{A}^{m} / \mathfrak{m}_{A}^{m+1}
$$

where $i$ is the natural inclusion ('complex' meaning that composites of successive maps are zero: note that each component of the complex has a distinguished zero element).

To define the complex it is only necessary to define $j$. Let $f\left(X_{i j}\right.$ : $0 \leq i \leq m-1,1 \leq j \leq n)=0$ be the equation defining $V$. Since $\operatorname{gr}_{A}\left(\mathrm{~m}_{A}\right)$ is without zero divisors, by Corollary 1(1) the coefficients of $f\left(X_{i j}\right)$ are polynomials in the $a_{l}$ 's of degree $m$. The proof of Proposition 1(2b) actually constructs a polynomial $f^{\#}\left(X_{i j}\right)$ in $S^{\circ}\left(\mathrm{m}_{A} / \mathrm{m}_{A}^{2}\right)\left[X_{i j}: 0 \leq i \leq\right.$ $m-1,1 \leq j \leq n], S^{*}\left(\mathrm{~m}_{A} / \mathrm{m}_{A}^{2}\right)$ denoting the symmetric algebra, whose image in $\operatorname{gr}\left(\mathrm{m}_{A}\right)[X]$ is $f\left(X_{i j}\right)$ under the canonical map. Denote by $a_{1}^{*}, \ldots, a_{n}^{\#}$ the unique liftings of $a_{1}, \ldots, a_{n}$ in $S^{\circ}\left(\mathrm{m}_{A} / \mathrm{m}_{A}^{2}\right)$. Let $z \in$ $T-\operatorname{sch}(\kappa, V)$ be given by $\left\{X_{i j} \rightarrow x_{i j} \in \kappa\right.$ for all $i, j$ with $\left.f\left(x_{\imath j}\right)=0\right\}$ and define $j(z)=f^{\#}\left(x_{i j}\right) \in S^{m}\left(\mathfrak{m}_{A} / \mathfrak{m}_{A}^{2}\right)$. Clearly $k \circ j=0$ since $k \circ j(z)=$ $k\left(f^{\#}\left(x_{i j}\right)\right)=f\left(x_{i j}\right)=0$. Note that the coefficients of $f^{\#}\left(X_{i j}\right)$, regarded as a polynomial in $a_{1}^{\#}, \ldots, a_{n}^{\#} \in S\left(\mathrm{~m}_{A} / \mathrm{m}_{A}^{2}\right)$ with coefficients in $\kappa\left(\mathfrak{m}_{A}\right)\left[X_{l j}\right.$ : all $\left.i j\right]$, are precisely the equations defining $V^{\prime}$. Thus $j(z)=0$ if and only if $z=i(y)$ for some $y \in T-\operatorname{sch}\left(\kappa, V^{\prime}\right)$ thus showing $j \circ i=0$, and $C^{\circ}$ is a complex. taking cohomology, we deduce $H^{0}\left(C^{\circ}\right)=H^{1}\left(C^{\circ}\right)=0$.

From Proposition 1, $\{\nu(\operatorname{discr} f)=s$ if and only if $\operatorname{ed}(f)=\operatorname{ed}(A)$, for every $f(X)$ in $\left.H_{g}(\operatorname{Spec} A)\right\}$ if and only if $i$ is surjective, thus if and only if $j$ is the zero map. We deduce:

Proposition 2. $H^{2}\left(C^{\circ}\right) \cong K_{m}$ if and only if $\{\boldsymbol{\nu}(\operatorname{discr} f(x))=s \Leftrightarrow$ $\operatorname{ed}(f(x))=\operatorname{ed}(A)$, for all $f(x)$ in $\left.H_{g}(\operatorname{Spec} A)\right\}$.

CoRollary 2. Suppose $f(X)$ has reduction $\Pi_{i} g_{i}(X)^{e_{i}} \bmod \mathfrak{m}_{A}$ which has one of the following:

(1) $f$ is totally ramified i.e. $\operatorname{deg} g_{i}=1$ for all $i$,

(2) $f$ is unramified i.e. $e_{i}=1$ for all $i$, 
(3) $K_{m}=0$ where $m=\operatorname{deg} f(x)$,

(4) $\operatorname{deg} f(X) \leq 3$,

then $\nu(\operatorname{discr} f(X))=\sum_{i}\left(e_{i}-1\right)\left[\kappa\left(\mathfrak{n}_{i}\right): \kappa\left(\mathfrak{m}_{A}\right)\right]$ if and only if $f$ is tamely ramified and $\operatorname{ed}(f(X))=\operatorname{ed}(A)$.

Proof. In any case, if $f(X)$ is wildly ramified the result follows so we assume $f$ is tamely ramified.

(1) Since $f$ is totally ramified, the equation $p\left(X_{i j}\right)$ defining $V$ is, by Corollary 1, a product $\Pi_{k} p_{k}\left(X_{i j}\right)$ of factors linear in the $X_{i j}$ 's and $a_{i}$ 's. Let $z \in T-\operatorname{sch}(\kappa, V)$ be given by $X_{i j} \rightarrow x_{i j} \in \kappa$ for all $i, j$, then $p\left(X_{i j}\right)=0$ implies $p_{k}\left(x_{i j}\right)=0$ for some $k$ since $\operatorname{gr}_{A}\left(\mathfrak{m}_{A}\right)$ has no zero divisors. Thus $p_{k}\left(x_{i j}\right)=0$ is a linear relation between the linearly independent $a_{i}$ 's so $j(z)=0$. Since $H^{1}\left(C^{\circ}\right)=0, z=i(y)$ for some $y \in T-\operatorname{sch}\left(\kappa, V^{\prime}\right)$ proving the corollary in view of Proposition 1.

(2) If $f$ is unramified, then obviously $\nu_{\mathrm{m}_{A}}(\operatorname{discr} f)=0$ since $f(X)=0$ has distinct roots $\bmod m_{A}$. Thus ed $(f(X))=\operatorname{ed}(A)$ by Theorem 1 .

(3) The Corollary follows immediately from Proposition 2.

(4) If $\operatorname{deg} f(X) \leq 3$ then the only possibilities are that $f$ is totally ramified or is unramified whence the result from (1) and (2).

Since resultants are 'universally' defined it easily follows from the proof of Proposition 1 that the subschemes $V, V^{\prime}$ of $T^{\prime}$ have a 'universal' construction in that they are induced from $\mathbf{Z}$-schemes independent of $T^{\prime}$ :

Proposition 3. Given non-negative integers $n, f_{1}, \ldots, f_{r}, e_{1}, \ldots, e_{r}$ there are affine $\mathbf{Z}$-schemes $Z, Z^{\prime}$ which are closed subschemes of $\mathbf{A}_{\mathbf{Z}}^{w}$, where $w=n+\sum_{i=1}^{r} f_{i}\left(e_{i} i+1\right)$, with the following property. For any local ring $A$ of embedding dimension $n$; any monic polynomial $g(X) \in A[X]$ with $g(X)$ $\equiv \prod_{i=1}^{r} g_{i}(X)^{e_{i}} \bmod m_{A}$ where $g_{i}(X)$ are distinct separable polynomials of degree $f_{l}$, there is a map $T^{\prime} \rightarrow \mathbf{A}_{\mathbf{Z}}^{w}$ so that $V=Z \times_{\mathbf{A}_{\mathbf{Z}}^{w}} T^{\prime}$ and $V^{\prime}=Z^{\prime} \times_{\mathbf{A}_{\mathbf{Z}}^{w}} T^{\prime}$.

EXAMPLE. We construct a quartic $f(X)$ over a 1-dimensional local ring $A$ with $f$ tamely ramified, $\operatorname{ed}(f)=\operatorname{ed}(A), \nu_{\mathrm{m}_{A}}(\operatorname{discr} f)=3$ and $s=$ $\sum_{i}\left(e_{i}-1\right)\left[\kappa\left(\mathfrak{n}_{i}\right): \kappa\left(\mathfrak{m}_{A}\right)\right]=2$.

Let $Q$ be the field of rational numbers and let $a_{1}, a_{2}$ be independent transcendentals over $Q$. Put $A=Q\left[a_{1}, a_{2}\right]_{\left\langle a_{1}, a_{2}\right\rangle} /\left\langle a_{1}^{2}+a_{2}^{2}\right\rangle$. Let $f(X)=$ $\left(X^{2}+1\right)^{2}+a_{2} X+a_{1}$ be a polynomial over $A$. 
We claim $A$ and $f(X)$ are our example.

For $f(X) \equiv\left(X^{2}+1\right)^{2} \bmod m_{A}$ so $f(X)$ is tamely ramified with ramification index 2 and $s=2$. The discriminant of $f(X)$ is

$\left(256 a_{1}^{3}-27 a_{2}^{4}+288 a_{1} a_{2}^{2}+256\left(a_{1}^{2}+a_{2}^{2}\right)\right) / 256=\left(-32 a_{1}^{3}-27 a_{1}^{4}\right) / 256$

so that $\nu_{\mathrm{m}_{A}}(\operatorname{discr} f)=3$.

$A$ is a 1-dimensional local ring of embedding dimension 2 and $\operatorname{gr}_{A}\left(\mathfrak{m}_{A}\right) \simeq Q\left[X_{1}, X_{2}\right] /\left\langle X_{1}^{2}+X_{2}^{2}\right\rangle$ has no zero divisors.

The maximal ideal of $A[X] /(f(X))$ is $\left\langle a_{1}, a_{2}, X^{2}+1\right\rangle=\left\langle a_{2}, X^{2}+\right.$ 1) since $a_{1}=-\left(X^{2}+1\right)^{2}-a_{2} X \in\left\langle a_{2}, X^{2}+1\right\rangle$ in $A[X] /(f(X))$. Thus $\operatorname{ed}(f(X))=2=\operatorname{ed}(A)$.

4. Applications. For the translation of Theorem 1 to schemes, we have:

THEOREM 2. Let $Y$ be a regular scheme and $\mathbf{P}_{Y}^{1}$ the projective line bundle over $Y[4]$. Let $X$ be a closed subscheme of $\mathbf{P}_{Y}^{1}$ so that for every irreducible component $X_{i}$ of $X$, the induced $f_{i}: X_{i} \rightarrow Y$ is dominating and finite and all residue field extensions are separable. Then:

(1) $X \rightarrow Y$ is flat;

(2) $X$ is regular and tamely ramified over $Y$ if and only if $\nu_{\mathrm{m}_{v}}\left(\mathfrak{d}_{X / Y, y}\right)=$ $\Sigma_{l}\left(e_{x_{t}}-1\right)\left[\kappa\left(m_{x_{t}}\right): \kappa\left(m_{y}\right)\right]$ for all points $x_{1}, \ldots, x_{n}$ in the fibre $f^{-1}(y)$ and for all points $y$ of $Y$.

By $\delta_{X / Y, y}$, we here mean the local discriminant of the finite free extension $O_{Y, y} \rightarrow \Gamma\left(X x_{Y} \operatorname{Spec} O_{Y, y}, O_{X x_{Y}} \operatorname{Spec} O_{Y, y}\right) ; e_{x_{1}}$ is defined similarly.

For the proof of the theorem, note that the question is local on $Y$ so we may assume $Y$ is affine. Moreover, we may replace $Y$ by Spec $O_{Y, y}$, by flat base change, and prove the theorem when $Y=\operatorname{Spec} A$ with $A$ a regular local ring. In this case, $\mathbf{P}_{A}^{1}=\operatorname{Proj} A\left[X_{0}, X_{1}\right]$ has every finite subset of points contained in an open affine subscheme isomorphic to $\operatorname{Spec} A[X]$; since condition (2) of the theorem is applied to such finite sets, we may assume $X$ is a closed subscheme of some $\operatorname{Spec} A[X]$. Let $X=\operatorname{Spec} A[X] / I$.

Let $I=q_{1} \cap q_{2} \cdots \cap q_{n}$ be the primary decomposition of $I$ in $A[X]$ and let $\mathfrak{p}_{i}=\sqrt{ } \mathfrak{a}_{l}, i=1, \ldots, n$, be the prime ideals associated to $\mathfrak{q}_{l}$. Then $\operatorname{Spec} A[X] /_{p_{l}} \rightarrow \operatorname{Spec} A$ is dominating and finite for each $i$. Therefore 
$\mathfrak{p}_{i} \cap A=\{0\}$ so height $\mathfrak{p}_{i}=1$ for each $i$. It easily follows that $\mathfrak{p}_{i}$ is a principal ideal, generated by $p_{i}(X)$, say where $p_{i}(X)$ is a non-constant polynomial in $A[X]$.

Since $\mathfrak{p}_{\imath} \cap A=\{0\}$ for all $i$, we have $\mathfrak{q}_{i}=\left\langle p_{i}(X)^{n_{t}}\right\rangle$ for some integers $n_{i}$. Therefore $\left\langle\Pi_{i} p_{i}(X)^{n_{i}}\right\rangle \subseteq I=\bigcap_{i=1}^{n} \mathfrak{a}_{i}$. In the fibre $A[X]$ $\otimes_{A} \operatorname{fract}(A), I$ and $\left\langle\Pi_{i} p_{l}(X)^{n_{i}}\right\rangle$ coincide since $A[X] \otimes_{A} \operatorname{fract}(A)$ is a principal ideal domain. It follows that for every $q(X) \in I$ there are $a, b \in A$ with $a q(X)=b p(X)$ where $p(X)=\Pi_{i} p_{i}(X)^{n_{i}}$.

But $A[X]\left\langle_{\left\langle p_{t}(X)\right\rangle}\right.$ is a finite $A$-module and, since $A$ is normal, Kronecker's Theorem [1] shows that $p_{l}(X)$ has invertible leading coefficient for all $i$; thus we may suppose $p(X), p_{1}(X), \ldots, p_{n}(X)$ are monic polynomials. Consequently, if $a q(X)=b p(X)$ then $q(X) \in\langle p(X)\rangle$ thus $I=\langle p(X)\rangle$ and so $X=\operatorname{Spec} A[X] /\langle p(X)\rangle$ where $p(X)$ is a monic polynomial; consequently $X \rightarrow Y$ is flat. The second part of the theorem now follows from Theorem 1 .

Corollary. With $f: X \rightarrow Y$ as in Theorem 2. Suppose that $\operatorname{Reg}(Y)$ is open (resp. contains a non-empty open set). Then the set of points $\{x \in X \mid X$ is regular and tamely ramified over $Y$ at every point of the fibre $\left.f^{-1} f(x)\right\}$ is open (resp. contains a non-empty open set).

Proof. By replacing $Y$ by a regular open subscheme we may assume $Y$ is regular. Now, $\nu_{m_{y}}\left(\mathfrak{D}_{X / Y, y}\right), \Sigma_{i}\left[\kappa\left(\mathfrak{m}_{x_{1}}\right): \kappa\left(\mathrm{m}_{y}\right)\right]$ are upper semi-continuous on $Y$ and $\Sigma_{i} e_{i}\left[\kappa\left(\mathfrak{m}_{x_{i}}\right): \kappa\left(\mathfrak{m}_{y}\right)\right]$ is locally constant; the corollary now follows.

\section{REFERENCES}

1. S. Abhyankar, Ramification Theoretic Methods in Algebraic Geometry, Annals of Math. Studies, Princeton University Press 1959.

2. A. Altman and S. Kleiman, Introduction to Grothendieck Duality Theory, Lecture Notes in Mathematics, 146, Springer, Heidelberg 1970.

3. J. P. Buhler, A note on tamely ramified polynomials, Pacific J. Math., 86 No. 2 (1980), 421-425.

4. R. Hartshorne, Algebraic Geometry, Graduate Texts in Mathematics, Springer-Verlag, New York-Heidelberg-Berlin 1977.

5. S. Lang, Algebra, Addison-Wesley 1971.

Received October 5, 1981.

UNIVERSITY COLLEGE

CARDIFF

South Glamorgan

WALES, GB 


\section{PACIFIC JOURNAL OF MATHEMATICS}

\section{EDITORS}

Donald BabbitT (Managing Editor)

University of California

Los Angeles, CA 90024

Hugo Rossi

University of Utah

Salt Lake City, UT 84112

C. C. Moore and Arthur Ogus

University of California

Berkeley, CA 94720
J. DugunduI

Department of Mathematics

University of Southern California

Los Angeles, CA 90089-1113

R. FinN and H. SAmelson

Stanford University

Stanford, CA 94305

\section{ASSOCIATE EDITORS}
R. ARENS
E. F. BECKENBACH
B. H. NEUMANN
F. WOLF
K. YosHIDA (1906-1982)

\section{SUPPORTING INSTITUTIONS}

UNIVERSITY OF ARIZONA

UNIVERSITY OF BRITISH COLUMBIA

CALIFORNIA INSTITUTE OF TECHNOLOGY

UNIVERSITY OF CALIFORNIA

MONTANA STATE UNIVERSITY

UNIVERSITY OF NEVADA, RENO

NEW MEXICO STATE UNIVERSITY

OREGON STATE UNIVERSITY
UNIVERSITY OF OREGON

UNIVERSITY OF SOUTHERN CALIFORNIA

STANFORD UNIVERSITY

UNIVERSITY OF HAWAII

UNIVERSITY OF TOKYO

UNIVERSITY OF UTAH

WASHINGTON STATE UNIVERSITY

UNIVERSITY OF WASHINGTON 


\section{Pacific Journal of Mathematics}

Vol. 107, No. $1 \quad$ January, 1983

John Kelly Beem and Phillip E. Parker, Klein-Gordon solvability and the

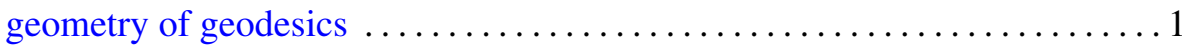

David Borwein and Amnon Jakimovski, Transformations of certain

sequences of random variables by generalized Hausdorff matrices ..... 15

Willy Brandal and Erol Barbut, Localizations of torsion theories . . . . . . . 227

John David Brillhart, Paul Erdős and Richard Patrick Morton, On sums

of Rudin-Shapiro coefficients. II ........................... 39

Martin Lloyd Brown, A note on tamely ramified extensions of rings $\ldots \ldots \ldots 71$

Chang P'ao Ch'ên, A generalization of the Gleason-Kahane-Żelazko

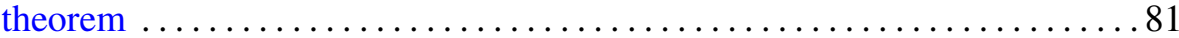

I. P. de Guzman, Annihilator alternative algebras $\ldots \ldots \ldots \ldots \ldots \ldots$. . . . 89

Ralph Jay De Laubenfels, Extensions of $d / d x$ that generate uniformly

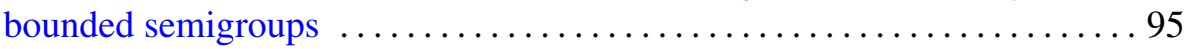

Patrick Ronald Halpin, Some Poincaré series related to identities of $2 \times 2$

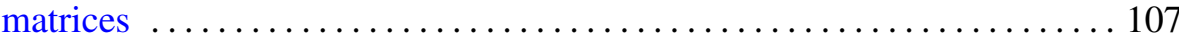

Fumio Hiai, Masanori Ohya and Makoto Tsukada, Sufficiency and

relative entropy in $*$-algebras with applications in quantum systems . . . 117

Dean Robert Hickerson, Splittings of finite groups $\ldots \ldots \ldots \ldots \ldots \ldots \ldots 14$

Jon Lee Johnson, Integral closure and generalized transforms in graded

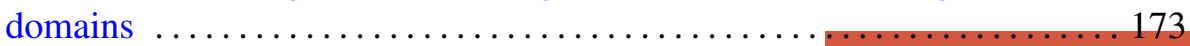

Maria Grazia Marinari, Francesco Odetti and Mario Raimondo, Affine

curves over an algebraically nonclosed field . ................. 179

Douglas Shelby Meadows, Explicit PL self-knottings and the structure of

PL homotopy complex projective spaces $\ldots \ldots \ldots \ldots \ldots \ldots \ldots \ldots \ldots$

Charles Kimbrough Megibben, III, Crawley's problem on the unique

$\omega$-elongation of $p$-groups is undecidable .................... 205

Mary Elizabeth Schaps, Versal determinantal deformations $\ldots \ldots \ldots \ldots 213$

Stephen Scheinberg, Gauthier's localization theorem on meromorphic

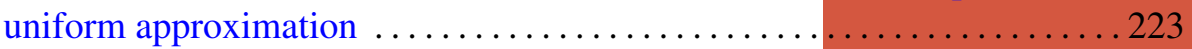

Peter Frederick Stiller, On the uniformization of certain curves . . . . . . . 229

Ernest Lester Stitzinger, Engel's theorem for a class of algebras . . . . . . . . 245

Emery Thomas, On the zeta function for function fields over $F_{p} \ldots \ldots \ldots 251$ 